\title{
Effect of stress and composition on the Raman spectra of etch-stop SiGeB layers
}

\author{
A. Pérez-Rodríguez, ${ }^{\text {a) }}$ A. Romano-Rodríguez, R. Cabezas, and J. R. Morante \\ EME, Department de Física Aplicada i Electronica, Universitat de Barcelona, \\ Avinguda Diagonal 645-647, 08028 Barcelona, Spain \\ T. Jawhari \\ Serveis Cientifico-Tècnics, Universitat de Barcelona, C. Solé i Sabarís 1-3, 08028 Barcelona, Spain \\ C. E. Hunt \\ Department of Electrical and Computer Engineering, University of California, Davis, California 95616
}

(Received 25 January 1996; accepted for publication 8 August 1996)

\begin{abstract}
$\mathrm{Si}_{1-(x+y)} \mathrm{Ge}_{x} \mathrm{~B}_{y}$ strained layers on $\mathrm{Si}(x \leqslant 3.4 \%, y \leqslant 0.4 \%)$ have been analyzed by Raman spectroscopy. Stress in the layers has not been observed to affect the Fano interaction parameters of the first-order Si-Si Raman line. These parameters have been determined in the range of $\mathrm{B}$ concentrations from $5 \times 10^{19}$ to $2 \times 10^{20} \mathrm{~cm}^{-3}$ and Ge fractions from $1 \%$ to $3.4 \%$. The observed shift in the spectra has been found to depend linearly on both the germanium and boron contents. These data have been correlated with the stress measured in the layers by mechanical wafer bow measurements. The dependence of the Raman shift on the germanium content and strain agrees with that previously reported for strained SiGe layers. According to these data, Raman spectroscopy appears as an interesting tool for the nondestructive assessment of stress and composition of these layers. (C) 1996 American Institute of Physics. [S0021-8979(96)00722-0]
\end{abstract}

\section{INTRODUCTION}

The most used way to obtain etch-stop layers in silicon is doping with a high concentration of boron (above $7 \times 10^{19}$ $\mathrm{cm}^{-3}$ ) the area where the etch has to be stopped. ${ }^{1-3}$ High-Bdoped $\mathrm{Si}$ shows very good etch selectivity in anisotropic etchants, such as $\mathrm{KOH}$, tetramethyl ammonium hydroxide (TMAH), or ethylenediaminepyrocatechol (EDP), and etchrate selectivities of the order of 5000 in relation to undoped Si can be achieved. ${ }^{4,5}$ However, in some cases the strain induced by the boron in the Si lattice constitutes a problem. This is especially critical for the obtention of silicon-oninsulator (SOI) structures by bonded and etch-back technology (known as BE SOI) ${ }^{6}$ In this case, stress in the $\mathrm{SiB}$ etch-stop layer induces the generation of misfit dislocations in the epitaxial Si layer grown on the etch-stop one, which will constitute the top Si layer in the final structure. To avoid this problem, stress in the etch-stop layer has to be compensated. For this, an interesting approach is the addition of germanium. ${ }^{4,7} \mathrm{SiGeB}$ epitaxial layers are, thus, interesting in $\mathrm{Si}$ technology because of their etch-stop properties.

These $\mathrm{Si}_{1-(x+y)} \mathrm{Ge}_{x} \mathrm{~B}_{y}$ layers are characterized by relatively low values of $x$ and $y$, B content being typically below $0.4 \%$ and Ge content below 3\%. For this low Ge content, Ge does not measurably alter the etching characteristics of the SiGeB layer, and etching selectivity is caused exclusively by the high boron concentration. This contrasts with the use of $\mathrm{SiGe}$ etch-stop layers, where Ge concentrations as high as $30 \%$ are required. ${ }^{5}$ Furthermore, expensive molecular-beamepitaxy (MBE) fabrication processing is required for the obtention of such layers, and the etch selectivity is much

${ }^{\text {a)} E l e c t r o n i c ~ m a i l: ~ p e r e z-r o @ i r i s 1 . f a e . u b . e s ~}$ smaller than that obtained with boron. Moreover, misfit generation occurs due to strain relaxation.

Nevertheless, in spite of the use of these SiGeB layers in the BE-SOI technology, an accurate and precise on-line control of the remaining stresses has not yet been achieved. In a general way, this might constitute a problem for the practical application of these layers. Remaining stress in the layers is usually determined by high-resolution $\mathrm{x}$-ray-diffraction (HRXRD) measurements; however, this technique presents problems for very low stress values, close to stress compensation. Moreover, for very thin layers, the HRXRD signal has to be deconvoluted from the substrate contribution.

Raman scattering is another well-known nondestructive technique which can be used for the stress assessment of these layers. The first-order Si Raman line (centered at about $520 \mathrm{~cm}^{-1}$ ) is sensitive to the presence of stress. ${ }^{8}$ Raman scattering has potential advantages over HRXRD, as in this case the measurement is performed in a surface region with a thickness determined by light absorption in the material. By selecting the excitation wavelength, a suited penetration depth can be achieved, avoiding the substrate contribution. Moreover, by the use of a micro-Raman spectrometer, high lateral resolution-below $1 \mu \mathrm{m}$-can be achieved in addition to depth resolution.

On the other hand, the analysis of the Raman spectra can also provide information on the composition of the $\mathrm{SiGeB}$ layer. The shape of the first-order Si Raman line is strongly affected by the presence of a high level of boron concentration $\left(\geqslant 10^{19} \mathrm{~cm}^{-3}\right) \cdot{ }^{9-11}$ This is due to the existence of a Fano-like interaction. The fitting of the Raman spectra allows us to determine the Fano parameters. The knowledge of these parameters gives a way to determine the boron concentration in the layer, as the main Fano parameters are strongly dependent on the boron content. 


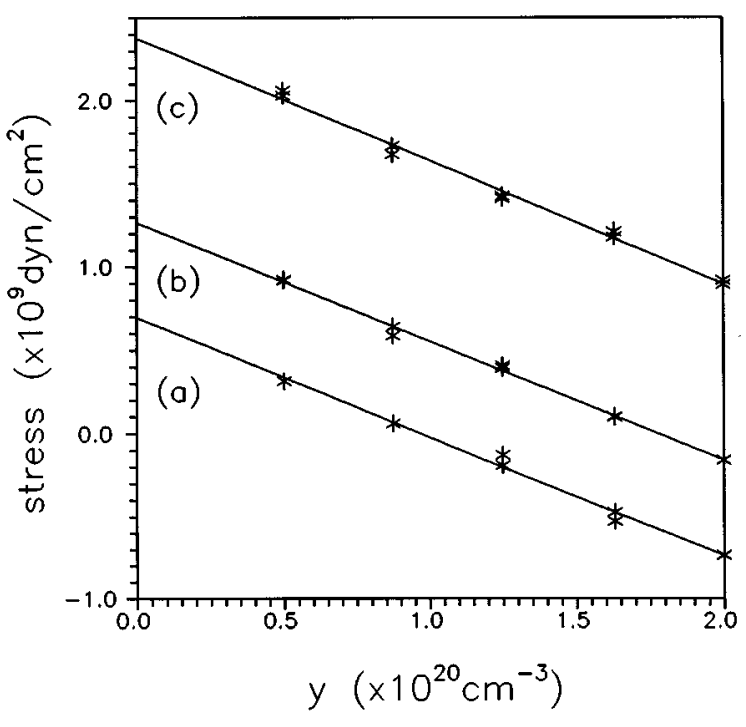

FIG. 1. Stress measured in the layers vs boron concentration for the different Ge contents: (a) $1.1 \%$, (b) $2.0 \%$, and (c) $3.3 \%$.

In this work, $\mathrm{Si}_{1-(x+y)} \mathrm{Ge}_{x} \mathrm{~B}_{y}$ strained layers with different compositions $(x \leqslant 3.4 \%, y \leqslant 0.4 \%)$ have been analyzed. The results obtained have been correlated with the stress in the layers determined by the change in the deflection curvature of the Si substrate and their composition. The fitting of the first-order $\mathrm{Si}-\mathrm{Si}$ Raman line measured on the layers with different stresses and compositions has been performed in order to analyze the dependence of the Fano interaction parameters on both composition and stress. The high crystalline quality of the $\mathrm{SiGeB}$ layers ${ }^{4}$ rules out any influence of structural defects on the Raman spectra. Then, the remaining shift in the spectra after their deconvolution according to the Fano interaction is determined by the stress and chemical composition of the layers. According to the obtained data, Raman spectroscopy is proposed for the nondestructive assessment of both stress and chemical composition in strained $\mathrm{Si}_{1-(x+y)} \mathrm{Ge}_{x} \mathrm{~B}_{y}$ layers, in the range of compositions $x \leqslant 3.4 \%, y \leqslant 0.4 \%$.

\section{EXPERIMENTAL DETAILS}

SiGeB layers were grown by low-temperature rapid thermal chemical-vapor deposition (RTCVD) on (100) Si wafers. The nominal thickness of these layers was $1.1 \mu \mathrm{m}$, and the range of $\mathrm{Ge}$ and $\mathrm{B}$ concentrations has been selected in agreement with those needed for etch-stop applications, including samples with compressive and tensile stress. Three different nominal $\mathrm{Ge}$ concentrations have been studied: $1.1 \%, 2.0 \%$, and $3.3 \%$. For each Ge concentration, layers with five different boron concentrations (between $5 \times 10^{19}$ and $2 \times 10^{20} \mathrm{~cm}^{-3}$ ) were grown.

The final Ge content and layer thickness has been determined by Rutherford backscattering (RBS). Stress in the samples was measured from the change in the deflection curvature of the $\mathrm{Si}$ substrate after deposition. Figure 1 shows the stress measured in the layers as a function of the boron content for the different Ge concentrations. As is shown, for a given Ge concentration the stress depends linearly on the
TABLE I. Thickness and composition of the different SiGeB layers.

\begin{tabular}{crcc}
\hline \hline Sample & $d(\mathrm{~nm})$ & {$[\mathrm{Ge}](\%)$} & {$[\mathrm{B}]\left(\mathrm{cm}^{-3}\right)$} \\
\hline 01 & 1100 & 1.1 & $5.0 \times 10^{19}$ \\
03 & 1100 & 1.1 & $8.75 \times 10^{19}$ \\
05 & 1100 & 1.2 & $1.25 \times 10^{20}$ \\
07 & 1125 & 1.1 & $1.63 \times 10^{20}$ \\
09 & 1110 & 1.1 & $2.0 \times 10^{20}$ \\
15 & 1085 & 2.0 & $5.0 \times 10^{19}$ \\
17 & 1085 & 2.0 & $8.75 \times 10^{19}$ \\
19 & 1085 & 2.0 & $1.25 \times 10^{20}$ \\
21 & 1085 & 2.0 & $1.63 \times 10^{20}$ \\
23 & 870 & 1.9 & $2.0 \times 10^{20}$ \\
29 & 1130 & 3.3 & $5.0 \times 10^{19}$ \\
31 & 1150 & 3.4 & $8.75 \times 10^{19}$ \\
33 & 1150 & 3.4 & $1.25 \times 10^{20}$ \\
35 & 1120 & 3.4 & $1.63 \times 10^{20}$ \\
37 & 870 & 3.3 & $2.0 \times 10^{20}$ \\
43 & 1185 & 1.0 & $5.0 \times 10^{16}$ \\
45 & 940 & 1.7 & $5.0 \times 10^{16}$ \\
47 & 900 & 2.7 & $5.0 \times 10^{16}$ \\
49 & 1120 & 1.0 & $5.0 \times 10^{17}$ \\
51 & 1120 & 1.7 & $5.0 \times 10^{17}$ \\
53 & 400 & 2.4 & $5.0 \times 10^{17}$ \\
\hline \hline
\end{tabular}

boron content. Moreover, stress also shows a linear dependency on the Ge content. This is the behavior which was to be expected, according to Vegard's law.

The different layers studied in this work are listed in Table I, together with their thickness and Ge and B content. As is shown in this table, layers with low boron concentration $\left(5 \times 10^{16}\right.$ and $\left.5 \times 10^{17} \mathrm{~cm}^{-3}\right)$ and Ge concentrations similar to those of the $\mathrm{SiGeB}$ ones have also been grown (layers 43-53).

Raman scattering measurements were performed using a Jobin-Yvon T64000 spectrometer. Measurements were made in backscattering geometry, being the excitation and light collection made through an Olympus metallographic microscope. The excitation of the samples was performed with the 457.9 and $488 \mathrm{~nm}$ lines from an $\mathrm{Ar}^{+}$laser. For these wavelengths, the penetration depth of scattered light in $\mathrm{Si}$ is estimated to be about 0.3 and $0.6 \mu \mathrm{m}$, respectively. According to this, the scattering volume is essentially located in the SiGeB layer. Moreover, the light spot on the sample is of about $0.6 \mu \mathrm{m}$. The excitation power has been kept below 500 $\mu \mathrm{W}$, which corresponds to power densities below 0.2 $\mathrm{MW} / \mathrm{cm}^{2}$. For these powers, no thermal effects were observed in the Raman spectra.

For all the samples the spectra have been compared with those measured in the same conditions on a single-crystal unprocessed Si sample (reference). The spectral resolution was $0.15 \mathrm{~cm}^{-1}$. When possible, the spectra have been fitted with a Lorentzian curve, which has allowed us to determine the peak position with an uncertainty of about $0.1 \mathrm{~cm}^{-1}$. For boron concentrations above $10^{19} \mathrm{~cm}^{-3}$, the spectra do not present a Lorentzian shape, due to the Fano interaction. In this case, the spectra have been fitted according to the Fano interaction model. 


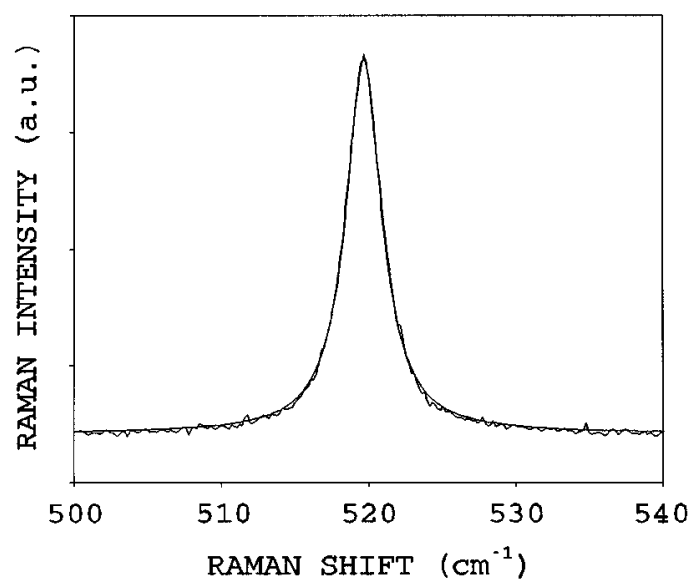

FIG. 2. First-order Si Raman line from sample 49, together with its fitting with a Lorentzian curve (excitation wavelength $457.9 \mathrm{~nm}$ ).

\section{EXPERIMENTAL RESULTS}

\section{A. SiGe layers with low boron concentration}

For the samples with the lowest boron concentrations $\left(5 \times 10^{16}\right.$ and $\left.5 \times 10^{17} \mathrm{~cm}^{-3}\right)$ the first-order Si Raman line has a Lorentzian shape, similar to the reference one. This can be seen in Fig. 2, where the spectrum from sample 49 is plotted, together with its fitting with a Lorentzian peak. For all these samples the peak appears at lower wave number than the reference one.

In Fig. 3 is plotted the shift of the Raman line (in relation to the reference one) as a function of the Ge concentration. This shift increases linearly with the Ge content, and does not depend on the B content or the excitation wavelength. The similarity between the spectra from the different wavelengths points out the homogeneity of the SiGe layers.

Different authors have reported the analysis of SiGe layers on $\mathrm{Si}$ as a function of the Ge concentration. ${ }^{12-17}$ According to them, the position of the Raman Si line depends on

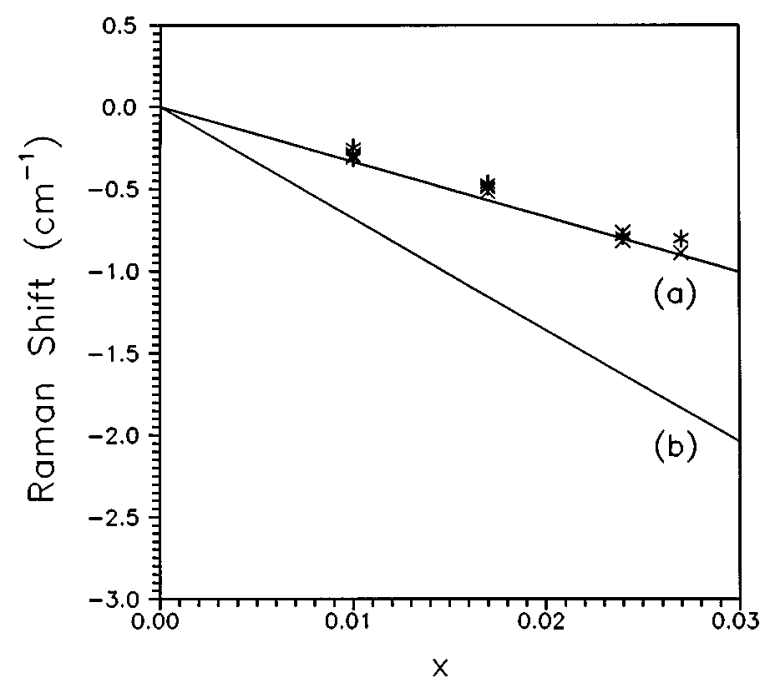

FIG. 3. Shift of the Raman line (in relation to the reference) from the samples with low boron concentration, vs Ge content. Excitation wavelength: (*) $457.9 \mathrm{~nm},(\times) 488 \mathrm{~nm}$. The solid lines are the shifts reported for (a) a fully stressed and (b) a fully relaxed SiGe layer (Refs. 12-17). both the layer composition and the strain in the layer. So, for a $\mathrm{Si}_{1-x} \mathrm{Ge}_{x}$ epitaxial layer on $\mathrm{Si}$, the $\mathrm{Si}$ first-order Raman line shifts toward lower frequencies in relation to that from bulk Si. This shift $\Delta \omega$ is given by ${ }^{17}$

$$
\Delta \omega\left(\mathrm{cm}^{-1}\right)=-68 x-830 \epsilon(x),
$$

where $\epsilon$ is the lattice mismatch strain between the $\mathrm{Si}_{1-x} \mathrm{Ge}_{x}$ layer and the $\mathrm{Si}$ substrate. For a relaxed (stress free) $\mathrm{Si}_{1-x} \mathrm{Ge}_{x}$ layer, the shift of the peak toward lower wave numbers is given by the first term in Eq. (1). This term represents the dependence of the vibrational mode on the composition of the layer. For a pseudomorphic (fully stressed) layer, the shift of the peak toward lower wave numbers decreases, due to the compressive stress in the layer. This dependence is given by the strain term in Eq. (1).

In Fig. 3 are also plotted the Raman shifts expected for a fully stressed and a fully relaxed layer, according to Eq. (1). For a partially relaxed layer, the shift of the Raman line is between those corresponding to the fully stressed and fully relaxed ones [lines (a) and (b) in the figure]. As is shown, the experimental data are close to those predicted for a fully stressed layer. This indicates that the grown layers are fully stressed.

In principle, stress in these layers will be larger than that in the layers with higher boron content, as boron will compensate the stress induced by the germanium atoms. Accordingly, the highest stress has been measured in samples 47 and 53 , being about $2.3 \times 10^{9} \mathrm{dyn} / \mathrm{cm}^{2}$. The fact that the layers with the highest stress from Table I are fully stressed strongly suggests all the other layers from this table to be fully stressed, as all of them have very similar thicknesses. This agrees with the linear relationship found between stress and composition, according to Fig. 1.

\section{B. SiGeB layers}

\section{Fano interaction}

As already indicated, the first-order Si Raman line depends on the concentration of boron, for concentrations $p \geqslant 10^{19} \mathrm{~cm}^{-3}$. $^{9-11}$ This is due to the existence of a continuum of electronic excitations produced by intervalenceband transitions whose energy overlaps with that of the phonon. This leads to a Fano-like interaction, which modifies the position and shape of the Raman line. In this case, the intensity of the line is given by

$$
I(\epsilon, q) \propto \frac{(q+\epsilon)^{2}}{1+\epsilon^{2}},
$$

with

$$
\epsilon=\frac{\left(\omega-\omega_{F}\right)}{\Gamma}
$$

and

$$
\omega_{F}=\omega_{p}+\delta \omega_{F}
$$

In these equations, $q$ is the asymmetry parameter, $\omega_{p}$ is the Raman phonon frequency in the absence of the Fano interaction, and $\delta \omega_{F}$ and $\Gamma$ are the shift- and width-related parameters, respectively. All the parameters strongly depend on 


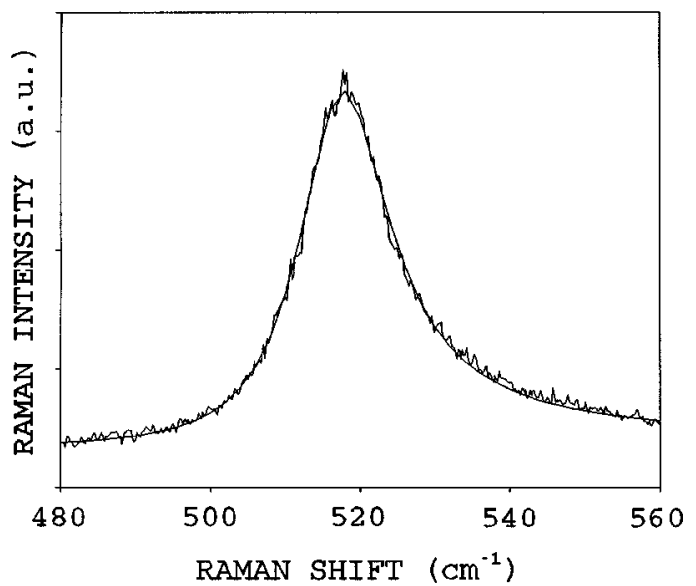

FIG. 4. First-order Raman spectrum from sample 07, and theoretical fitting according to the Fano interaction (excitation wavelength $457.9 \mathrm{~nm}$ ).

the concentration of holes, which is given by the boron content. In addition to them, Cerdeira and co-workers ${ }^{9}$ defined a fifth parameter $\eta$, which does not depend on the carrier concentration. This is given by

$$
\eta=\left(\Gamma^{3} q^{2}\right)^{1 / 5} \text {. }
$$

Moreover, these parameters show different behaviors in relation to the excitation wavelength: both $\Gamma$ and $\delta \omega_{F}$ do not depend on the excitation wavelength, while $q$ and $\eta$ do change with the excitation wavelength. This behavior has been interpreted theoretically in terms of the detailed valence-band structure of $\mathrm{Si}$ and the resonant characteristics of the vibronic and electronic Raman scattering. ${ }^{9}$

\section{Fitting of experimental spectra}

The experimental spectra measured in the layers with boron content higher than $10^{19} \mathrm{~cm}^{-3}$ have been fitted with expression (2), according to the Fano interaction. In Fig. 4 is shown the spectrum measured from sample 07, together with its fitting. From the fitting of these spectra, the values of the different Fano parameters have been determined as a function of the B and Ge content, as well as of the excitation wavelength.

These values are plotted in Figs. 5(a) and 5(b), as a function of the boron concentration. As is shown in these figures, the parameters $q$ and $\Gamma$ obtained from spectra measured at the same excitation wavelength in samples with the same B content and different Ge content are very similar, being in almost all the cases changes in these parameters below $6 \%$ of their value. This uncertainty is similar to the one reported in previous works, ${ }^{9}$ and indicates that, in the range of Ge content of these layers, the Fano interaction is not affected by the stress in the layers. This is in agreement with the results of Cerdeira and co-workers, ${ }^{9}$ which observed low changes of the Fano parameters for uniaxial stresses below $2 \times 10^{9} \mathrm{dyn} / \mathrm{cm}^{2}$.

In Figs. 5(a) and 5(b) are plotted the parameters obtained from the spectra measured in the different samples, using excitation wavelengths of 457.9 and $488 \mathrm{~nm}$. As is shown, very similar values of $\Gamma$ are obtained at both excitation
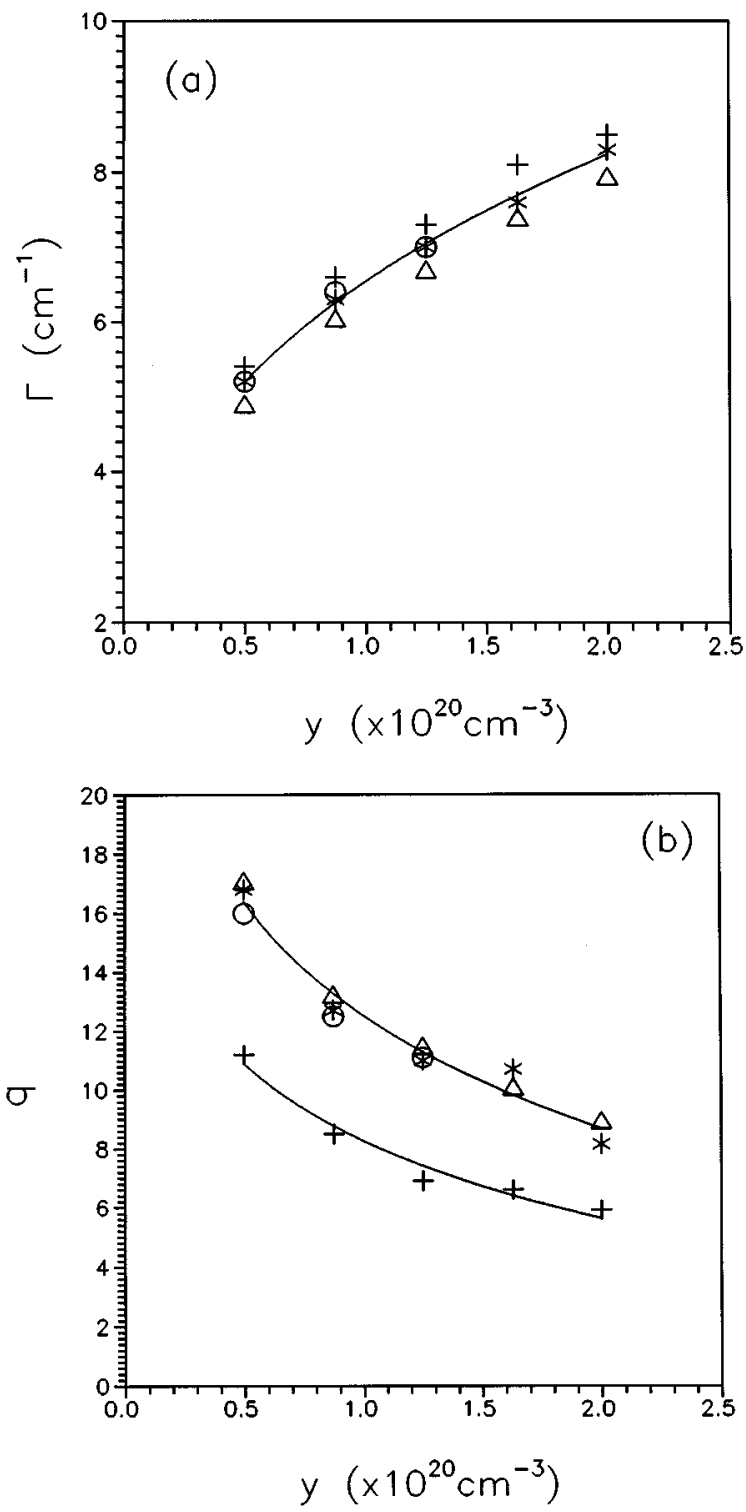

FIG. 5. (a) $\Gamma$ and (b) $q$ parameters vs boron concentration from the spectra measured in the samples with different $\mathrm{Ge}$ contents and excitation wavelengths. Ge content: $(\triangle) 1.1 \%,(*) 2.0 \%$, and $(\bigcirc,+) 3.3 \%$. Excitation wavelength: $(\triangle, *, \bigcirc) 457.9 \mathrm{~nm}$ and $(+) 488 \mathrm{~nm}$. Solid lines in these figures are plotted as a guide for the eye.

wavelengths. In addition, the value of $\eta$ does not change for the different boron concentrations, depending only on the excitation wavelength. The values obtained are $\eta=8.4 \pm 0.3$ $\mathrm{cm}^{-3 / 5}$ and $\eta=7.3 \pm 0.2 \mathrm{~cm}^{-3 / 5}$ for the used excitation wavelengths of 457.9 and $488 \mathrm{~nm}$, respectively. The agreement of these results with the theoretical analysis and previous data already reported ${ }^{9}$ gives support to the performed fittings.

The average values of the different parameters plotted in Figs. 5(a) and 5(b) are indicated in Table II. This table summarizes the results obtained on the dependence of the $q$ and $\Gamma$ parameters on the boron content. According to the dispersion of the obtained values, their uncertainty is below $10 \%$.

In Fig. 6 are plotted the values of $\Delta \omega_{F}=\omega_{F}-\omega_{0}$ [where $\omega_{0}$ is the frequency of the Raman line from the reference, not processed, Si sample and $\omega_{F}$ is defined in expression (4)] versus the boron content, for the different Ge contents. As is 
TABLE II. Average values of the Fano parameters from Figs. 5(a) and 5(b).

\begin{tabular}{|c|c|c|c|}
\hline \multirow{2}{*}{$\begin{array}{c}{[\mathrm{B}]} \\
\left(\mathrm{cm}^{-3}\right)\end{array}$} & \multicolumn{2}{|c|}{$q$} & \multirow{2}{*}{$\begin{array}{c}\Gamma \\
\left(\mathrm{cm}^{-1}\right)\end{array}$} \\
\hline & $(457.9 \mathrm{~nm})$ & $(488 \mathrm{~nm})$ & \\
\hline $5 \times 10^{19}$ & 16.6 & 11.2 & 5.3 \\
\hline $8.75 \times 10^{19}$ & 12.8 & 8.5 & 6.3 \\
\hline $1.25 \times 10^{20}$ & 11.2 & 6.9 & 7.0 \\
\hline $1.63 \times 10^{20}$ & 10.4 & 6.6 & 7.7 \\
\hline $2.0 \times 10^{20}$ & 8.55 & 5.9 & 8.25 \\
\hline
\end{tabular}

shown, the shift $\Delta \omega_{F}$ depends linearly on both the Ge and B contents. The linear regression analysis of these data gives the relationship

$$
\Delta \omega_{F}\left(\mathrm{~cm}^{-1}\right)=-0.6-37 x-958 y,
$$

where $x$ and $y$ are the $\mathrm{Ge}$ and $\mathrm{B}$ atomic fractions, respectively. In this figure are also plotted the Raman shifts given by this expression (dashed lines). The difference between the experimental values and those from expression (6) is below the uncertainty of these measurements (of about $0.2 \mathrm{~cm}^{-1}$ ).

\section{DISCUSSION}

According to Eq. (4), the Raman shift $\Delta \omega_{F}$ can be expressed as

$$
\Delta \omega_{F}=\omega_{F}-\omega_{0}=\left(\delta \omega_{F}+\omega_{p}\right)-\omega_{0}=\delta \omega_{F}+\Delta \omega_{p},
$$

where $\Delta \omega_{p}$ is the difference between the frequency of the Raman line in the absence of the Fano interaction $\omega_{p}$ and the frequency of the Raman line from the reference Si sample $\omega_{0}$. In principle, and in agreement with the behavior reported from $\mathrm{SiGe}$ and SiGeC layers, ${ }^{17} \Delta \omega_{p}$ is expected to depend linearly on the Ge and $\mathrm{B}$ contents and on the strain in the layer. According to expression (1), the Raman shift can be approximated by

$\Delta \omega_{p}(x, y)\left(\mathrm{cm}^{-1}\right)=B(y)-68 x-830 \epsilon(x, y)$,

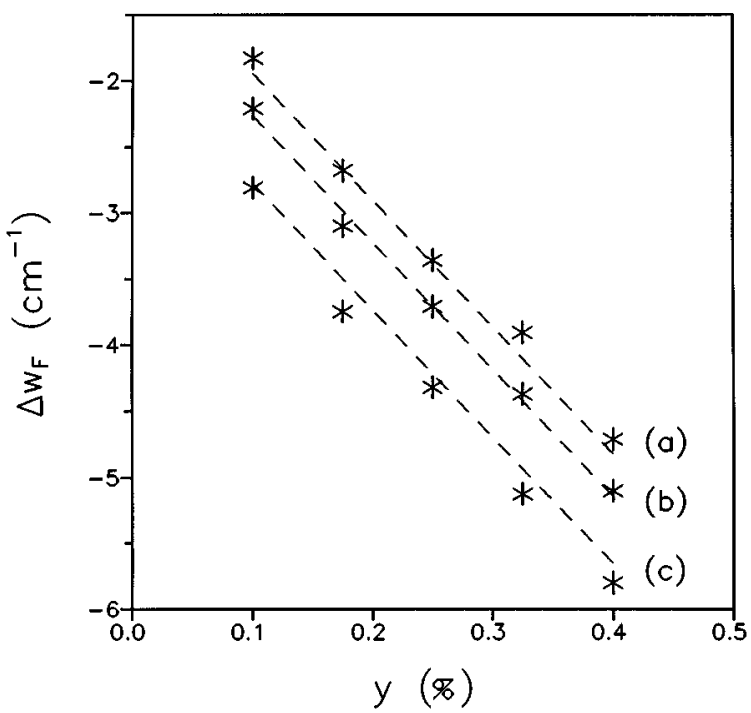

FIG. 6. $\Delta \omega_{F}=\omega_{F}-\omega_{0}$ vs boron atomic fraction from the different measured samples. Ge content: (a) $1.1 \%$, (b) $2.0 \%$, and (c) $3.3 \%$. Dashed lines are obtained from the linear regression analysis of the experimental data. and, therefore,

$\Delta \omega_{F}(x, y)\left(\mathrm{cm}^{-1}\right)=\delta \omega_{F}+B(y)-68 x-830 \epsilon(x, y)$,

where the second and third terms from Eq. (9) correspond to the compositional dependence of the mode without strain, and $\epsilon$ is the strain in the layer. In this expression we have assumed that the coefficient of the strain term in the Raman shift remains unchanged upon $\mathrm{B}$ addition. This assumption is reasonable, as this coefficient does not depend on the $\mathrm{Ge}$ content for $\mathrm{Si}_{1-x} \mathrm{Ge}_{x}$ layers. ${ }^{17}$

On the other hand, for a $\mathrm{SiGeB}$ strained layer the strain is given by both the $\mathrm{Ge}$ and $\mathrm{B}$ atomic fractions. Assuming a biaxial stress in the layer determined by the lattice mismatched strain in the $\mathrm{SiGeB} / \mathrm{Si}$ heterostructure, ${ }^{8}$ and the elastic constants of $\mathrm{Si}$, the linear relationship found between the stress and the chemical composition (shown in Fig. 1) gives the following dependency of the strain $\epsilon$ on the Ge and B contents,

$$
\epsilon=-0.038 x+0.200 y \text {. }
$$

Taking into account this expression, Eq. (9) becomes

$$
\Delta \omega_{F}=\delta \omega_{F}+B(y)-36.5 x-166 y .
$$

We have to remark the good agreement between the third term in this expression and the second term in expression (6) (better than 2\%), which was obtained from the linear fitting of the experimental data. According to this, the dependence of the Raman shift on the Ge atomic fraction $x$ is not affected by the presence of the boron atoms.

The experimental data plotted in Fig. 6 also show a linear dependence of the Raman shift on the B atomic fraction $y$. This is the behavior to be expected for the boron-related composition and strain terms in $\Delta \omega_{p}$. Moreover, in the range of values of boron concentration in the studied samples, the data reported by previous authors also suggest a linear dependence of the Fano-induced shift $\delta \omega_{F}$ on the boron content; ${ }^{9}$ however, the origin of the offset of $-0.6 \mathrm{~cm}^{-1}$ in expression (6) is not clear. The fitting of the experimental data from Fig. 6 requires the presence of this term. This could be related to the fact that $\delta \omega_{F}$ is not completely proportional to the boron atomic fraction $y$, and it can be approximated by a linear dependence only in a certain range of values of $y$. Anyway, further analysis is required to clarify the origin of this term, which could also be related to deviations from the linear behavior of the dependence of the mode on the boron composition. Nevertheless, the experimental results reported in this work on wafer bow and Raman scattering measurements provide a way to estimate the stress and chemical composition of the $\mathrm{SiGeB}$ strained layers from the Raman data, in the range of compositions studied. So, the fitting of the Raman spectra according to the Fano interaction model gives the values of the $q$ and $\Gamma$ parameters. The comparison of these values with those shown in Table II allows us to estimate the boron concentration. Then, from expression (6) the Ge content can be determined. Finally, once both $\mathrm{B}$ and $\mathrm{Ge}$ contents are known, stress in the layer can be determined, according to the linear behavior shown in Fig. 1. 


\section{CONCLUSIONS}

In summary, the analysis by Raman spectroscopy of $\mathrm{Si}_{1-(x+y)} \mathrm{Ge}_{x} \mathrm{~B}_{y}$ strained layers on $\mathrm{Si}$ has been performed. The previous analysis of SiGe layers with low boron concentration corroborates the absence of stress relaxation in these layers. The fitting of the first-order Si-Si Raman line has allowed us to obtain the values of the Fano parameters $q, \Gamma$, $\eta$, and $\omega_{F}$ for boron concentrations in the range between $5 \times 10^{19}$ and $2 \times 10^{20} \mathrm{~cm}^{-3}$. The parameters $q, \Gamma$, and $\eta$ have been found to be independent of the stress in the layer, depending only on the boron concentration and/or excitation wavelength. The Raman shift $\Delta \omega_{F}$ has been found to depend linearly on both the Ge and B contents. Moreover, the dependence of the Raman shift on the Ge content and strain is not affected by the presence of the B atoms.

These results indicate the ability of Raman spectroscopy for the independent stress and composition assessment of SiGeB strained layers in the range of compositions studied. For a given SiGeB layer, the comparison of the Fano-related parameters with those reported in Table II allows the boron concentration to be estimated. Once this is known, the germanium concentration can be determined from expression (6). The knowledge of both magnitudes (germanium and boron content) allows us to determine the stress in the layer, according to the linear behavior shown in Fig. 1.

\section{ACKNOWLEDGMENTS}

The authors wish to thank Dr. McDonald Robinson of Lawrence Semiconductor Research Laboratory in Tempe,
AZ for the mechanical wafer bow measurements and constructive comments, Dr. Leonard C. Feldman and his assistants Netzer Moriya and W. King of ATT Bell Labs for providing the RBS data, and the Calibration Standards Group at NIST for providing samples for calibration.

${ }^{1}$ H. A. Waggener, Bell Syst. Tech. J. 49, 473 (1970).

${ }^{2}$ H. Seidel, L. Csepregi, A. Heuberger, and H. Baumgartel, J. Electrochem. Soc. 137, 3626 (1990).

${ }^{3}$ N. F. Raley, Y. Sugiyama, and T. Van Duzer, J. Electrochem. Soc. 131, 161 (1984).

${ }^{4}$ C. E. Hunt, G. V. Rouse, C. Harendt, and M. C. Green, in Proceedings of the 1990 IEEE SOS/SOI Technical Conference, Key West, FL, 1990, p. 145.

${ }^{5}$ D. Feijóo, J. C. Bean, L. J. Peticolas, L. C. Feldman, and W. C. Liang, J. Electron. Mater. 23, 493 (1994).

${ }^{6}$ C. Harendt, C. E. Hunt, W. Appel, H. G. Graf, B. Höfflinger, and E. Penteker, J. Electron. Mater. 20, 267 (1991).

${ }^{7}$ C. A. Desmond, C. E. Hunt, and S. N. Farrens, J. Electrochem. Soc. 141, 178 (1994).

${ }^{8}$ E. Anastassakis, in Physical Problems in Microelectronics, edited by J. Kassabov, Proceedings of the 4th International School ISPPM, Varna, Bulgaria (1985), p. 128

${ }^{9}$ F. Cerdeira, T. A. Fjeldly, and M. Cardona, Phys. Rev. B 8, 4734 (1973).

${ }^{10}$ R. Beserman and T. Bernstein, J. Appl. Phys. 48, 1548 (1977).

${ }^{11}$ H. Engstrom and J. B. Bates, J. Appl. Phys. 50, 2921 (1979).

${ }^{12}$ F. Lu, C. H. Perry, F. Namavar, N. L. Rowell, and R. A. Soret, Appl. Phys. Lett. 63, 9 (1993).

${ }^{13}$ J. C. Tsang, P. M. Mooney, F. Dacol, and J. O. Chu, J. Appl. Phys. 75, 8098 (1994).

${ }^{14}$ M. I. Alonso and K. Winer, Phys. Rev. B 39, 10056 (1989).

${ }^{15}$ D. J. Lockwood and J. M. Baribeau, Phys. Rev. B 45, 8565 (1992).

${ }^{16}$ B. Dietrich, E. Bugiel, J. Klatt, G. Lippert, T. Morgenstern, H. J. Osten, and P. Zaumseil, J. Appl. Phys. 74, 3177 (1993).

${ }^{17}$ J. Menéndez, P. Gopalan, G. S. Spencer, N. Cave, and J. W. Strane, Appl. Phys. Lett. 66, 1160 (1995). 